\title{
Cuidado às pessoas com deficiência auditiva sob o olhar do enfermeiro de família
}

\author{
Care for People with Hearing Impairment from the Perspective of the Family Nurse
}

Cuidado de las personas con discapacidad auditiva desde la perspectiva del enfermero de familia

\author{
Anna Keylla da Silva dos Santos ${ }^{1}$, ORCID 0000-0001-6363-2003 \\ Lucas Portella Silva Santos ${ }^{2}$, ORCID 0000-0002-1536-7126 \\ Magda Guimarães de Araujo Faria ${ }^{3}$, ORCID - 0000-0002-4191-5480 \\ Paula Soares Brandão ${ }^{4}$, ORCID 0000-0001-6831-0038 \\ Priscila Cristina da Silva Thiengo ${ }^{5}$, ORCID 0000-0003-0840-4838 \\ Luciana Valadão Vasconcelos Alves ${ }^{6}$, ORCID 0000-0003-2159-543X \\ ${ }^{1}$ Universidade Federal Fluminense, Brasil \\ 2345 Universidade do Estado do Rio de Janeiro, Brasil \\ ${ }^{6}$ Insituto Federal de Educação, Ciência e Tecnologia, Brasil
}

Resumo: Objetivo: Descrever aspectos do cuidado às pessoas com deficiência auditiva a partir da avaliação de enfermeiros atuantes na saúde da família. Método: Estudo qualitativo, descritivo, exploratório do tipo piloto. A população inicial do estudo era de 53 indivíduos, entretanto, a amostra foi de 37 enfermeiros entre egressos e alunos do Curso de Especialização em Enfermagem em Saúde da Família, na Modalidade Residência. Para o desenvolvimento da investigação, o projeto proposto foi avaliado por um Comitê de Ética em Pesquisa e aprovado sob número de parecer 2.543.278. Resultados: 62,16\% dos participantes já atenderam pacientes com deficiência auditiva; 92\% relataram que este atendimento não foi totalmente eficaz e apenas $11 \%$ mencionaram a existência de recursos que garantiam um atendimento adequado; $27 \%$ dos participantes não se sentiam aptos para prestar assistência a este público e todos perceberam a necessidade de qualificação para efetuar o cuidado à pessoa com deficiência auditiva, sendo que a estratégia mais citada foi a incorporação de conteúdos na graduação. Conclusão: Esta análise foi responsável por um diagnóstico conclusivo de insegurança, incapacidade de atendimento e despreparo da própria unidade de saúde, o que aponta para a necessidade de construção e reformulação de políticas públicas de educação permanente e de manutenção de ambientes institucionais de saúde amigáveis que possam garantir a estruturação de um sistema de atendimento resolutivo à esta população.

Palavras-chave: cuidados de enfermagem; pessoas com deficiência auditiva; estratégia saúde da família; atenção primária à saúde. 
Abstract: Objective: To describe aspects of care for people with hearing impairment from the assessment of nurses working in family health. Method: Qualitative, descriptive, exploratory pilot study. The initial population of the study was 53 individuals, however, the sample consisted of 37 nurses, including graduates and students of the Specialization Course in Nursing in Family Health, in the Residence Modality. For the development of the investigation, the proposed project was evaluated by a Research Ethics Committee and approved under opinion number 2.543.278. Results: $62.16 \%$ of participants have seen patients with hearing impairment; $92 \%$ reported that this service was not fully effective and only $11 \%$ mentioned the existence of resources that ensure adequate care; $27 \%$ of participants did not feel able to provide assistance to this audience and all perceived the need for qualification to provide care to people with hearing impairment, and the most cited strategy was the incorporation of content in graduation. Conclusion: This analysis was responsible for a conclusive diagnosis of insecurity, incapacity of care and unpreparedness of the health unit itself, which points to the need to build and reformulate public policies for permanent education and maintenance of friendly institutional health environments that guarantee the structuring of a resolute service system for this population.

Keywords: nursing care; persons with hearing impairments; family health strategy; primary health care.

Resumen: Objetivo: Describir aspectos del cuidado a personas con discapacidad auditiva a partir de la evaluación de enfermeros que trabajan en salud familiar. Método: Estudio piloto exploratorio, cualitativo, descriptivo. La población inicial del estudio fue de 53 individuos, sin embargo, la muestra estuvo conformada por 37 enfermeros, entre egresados y estudiantes del Curso de Especialización en Enfermería en Salud de la Familia, en la Modalidad Residencia. Para el desarrollo de la investigación, el proyecto propuesto fue evaluado por un Comité de Ética en Investigación y aprobado bajo el dictamen número 2.543.278. Resultados: $62,16 \%$ de los participantes ha atendido a pacientes con discapacidad auditiva; El 92\% informó que este servicio no era plenamente efectivo y solo el 11\% mencionó la existencia de recursos que aseguren una atención adecuada; $27 \%$ de los participantes no se sintió capaz de brindar asistencia a este público y todos percibieron la necesidad de capacitación para brindar atención a personas con discapacidad auditiva, y la estrategia más citada fue la incorporación de contenidos en la graduación. Conclusión: Este análisis fue responsable de un diagnóstico concluyente de inseguridad, incapacidad de atención y falta de preparación de la propia unidad de salud, lo que apunta a la necesidad de construir y reformular políticas públicas de educación permanente y mantenimiento de ambientes institucionales de salud amigables que garanticen la estructuración de un sistema de servicio decidido para esta población.

Palabras claves: atención de enfermería; personas con deficiencia auditiva; estrategia de salud familiar; atención primaria de salud.

Recebido: 31/12/2020

Aceito: 05/08/2021 
Como citar:

Dos Santos AKDS, Santos LPS, Faria MGDA, Brandão PS, Thiengo PCDS, Alves LVV. Cuidado às pessoas com deficiência auditiva sob o olhar do enfermeiro de família. Enfermería: Cuidados Humanizados. 2021;10(2):89-101. DOI: 10.22235/ech.v10i2.2419

Correspondência: Magda Guimarães de Araujo Faria. E-mail: magda.faria@live.com

\section{Introdução}

Esta pesquisa possui como objetivo descrever aspectos do cuidado às pessoas com deficiência auditiva a partir da avaliação de enfermeiros atuantes na saúde da família. Ressalta-se que ainda são muitas as dificuldades voltadas ao atendimento deste público-alvo e dentre os principais fatores observados encontra-se o despreparo do profissional de saúde para a comunicação e realização de atividades humanizadas de cuidado à estes usuários. ${ }^{(1)}$

No contexto mundial, o número de pessoas acometidas por deficiências auditivas incapacitantes chega a $5 \%$, ou seja, quase 470 milhões de pessoas, porém a expectativa é que esse número chegue a 900 milhões em 2050 (2) $^{(20}$ cenário brasileiro, o censo demográfico $^{(3)}$ aponta a existência de 45,6 milhões de brasileiros o acometimento de pelo menos um tipo de deficiência, o que corresponde a 23,9 \% da população total, contudo, o percentual de indivíduos com funcionalidade comprometida para a vida em sociedade é de $8,2 \%$, sendo que $1,1 \%$ apresenta total disfuncionalidade auditiva. ${ }^{(4)}$

A pessoa com deficiência auditiva enfrenta inúmeras dificuldades de acesso aos serviços de saúde, visto que a audição é um sentido fundamental para obtenção e uso da linguagem, interferindo pontualmente no recebimento de informações por meio oral, além da manutenção do vínculo com o profissional de saúde e na compreensão sobre o processo saúde-doença. ${ }^{(5)}$ Ademais, ressalta-se o diagnóstico situacional que, devido a dificuldades impostas pelas adaptação social às suas limitações, comumente apresentam níveis de escolaridade e renda mais baixos se comparados à população não-surda. ${ }^{(6)}$

Neste sentido, o programa de Residência em ESF é um grande aliado, visto que um dos papeis fundamentais de qualificações desta natureza é de estimular as práticas de educação permanente em saúde, que por sua vez, devem ser baseadas na vivência prática do próprio profissional. ${ }^{(7,8)}$ Contudo, é necessário enfatizar que a capacitação para o cuidado de grupos vulneráveis como as pessoas com deficiência, nem sempre é contemplada na residência, sobretudo pela ausência de profissionais preparados para a cooperação na qualificação dos discentes.

Cumpre observar que a Política Nacional de Atenção Básica do Brasil prevê infraestrutura para o atendimento de pessoas com deficiência mas não ressalta a formação de recursos humanos para o cuidado, o que corrobora para um possível afastamento entre indivíduos com necessidades específicas de atenção e serviços de saúde. ${ }^{(9)}$

As dificuldades no atendimento, no cuidado e na construção do vínculo com indivíduos com deficiência auditiva é percebida em várias partes do mundo. Nos Estados Unidos, observou-se que usuários surdos possuem uma chance três vezes menor de conseguir agendar uma consulta em relação a indivíduos não surdos. ${ }^{(10)}$ Já em estudo realizado na Inglaterra, $44 \%$ dos usuários surdos considerou o atendimento na atenção primária insuficiente para suas demandas. ${ }^{(11)}$

A justificativa desta pesquisa ancora-se na necessidade de estudos que abordem o cuidado de pessoas com deficiência auditiva e a manutenção da comunicação com estes 
indivíduos, já que estas práticas representam um grande desafio para os profissionais da saúde e para o atendimento humanizado. Enfatiza-se ainda, que o enfermeiro precisa inserirse, ativamente, na equipe de reabilitação, promovendo educação em saúde, reabilitação e autocuidado, além de desenvolver consciência transitiva crítica para facilitar a inclusão social.

Não obstante a isso, ressalta-se a escassez de estudos que abordam a temática. $\mathrm{O}$ cuidado a indivíduos com deficiência auditiva ainda é pouco explorado na literatura cientifica e, quando se trata do envolvimento de enfermeiros residentes, esta torna-se totalmente inexistente.

Destaca-se ainda que o enfermeiro atuante na ESF tem em âmbito de prática, a necessidade de articulação de dimensões humanísticas de cuidado que envolvem desde o acolhimento à atividades gerenciais e a implementação da assistência de Enfermagem. ${ }^{(12)}$ Além disso, o processo eficiente de comunicação entre enfermeiro, paciente e família viabiliza uma assistência humanística e personalizada de acordo com as necessidades da pessoa atendida. ${ }^{(13)}$

\section{Métodos}

Estudo qualitativo, descritivo, exploratório do tipo piloto. O cenário compreendeu um curso de especialização em enfermagem em saúde da família na modalidade residência de uma instituição pública de ensino superior do estado do Rio de Janeiro/Brasil. O período de coleta esteve compreendido entre os meses de julho e setembro de 2018.

A população do estudo foi de 53 indivíduos entre egressos e alunos do Curso de Especialização em Enfermagem em Saúde da Família na Modalidade Residência. O critério de inclusão dos participantes foi a participação prévia em uma disciplina realizada no segundo ano da residência, intitulada "Clínica de Atenção Primária: Indivíduo, Família e Comunidade na perspectiva das linhas de cuidado I". Nesta disciplina é apresentado o conteúdo de sistematização da Assistência de Enfermagem, abordagem familiar e consultas de enfermagem aos indivíduos e suas famílias em todos os ciclos de vida, incluindo os grupos de vulnerabilidade como as pessoas com deficiência.

A amostra por conveniência foi contemplada com 37 participantes entre 16 enfermeiros egressos e 21 enfermeiros residentes do Curso de Especialização em Enfermagem em Saúde da Família na Modalidade Residência, de acordo com a disponibilidade de tempo e interesse de participar da pesquisa.

Para a obtenção dos dados, foi elaborado um questionário online com perguntas abertas e fechadas baseado nas diretrizes da Política Nacional de Saúde da Pessoa com Deficiência. ${ }^{(14)}$ Esse questionário foi constituído pelos pesquisadores e foi dividido em três partes. A primeira parte apresenta identificação do participante, formada por quatro questões. A segunda parte possui cinco questões que detalha o perfil dos profissionais. Já a terceira parte possui vinte questões, com o objetivo de buscar informações sobre o cuidado a pessoa com deficiência auditiva. O instrumento de coleta de dados foi construído através da ferramenta de formulários do Google Forms e enviado por e-mail para cada participante com anuência da coordenação pedagógica do curso.

As respostas foram organizadas em formato de planilhas do Microsoft Office Excel®. As variáveis foram traçadas com base em informações que transcorrem desde as características sociodemográficas até mesmo o perfil de atendimento destes profissionais. Cita-se como variáveis de destaque nesta investigação: idade, sexo, vinculação, etnia, 
instituição de formação, preparo da unidade para o atendimento, preparo pessoal para o atendimento.

Para análise foi utilizada a estatística descritiva simples com o apoio do programa IBM SPSS Statistics® 20 para as questões fechadas, o que auxiliou na construção de tabelas bem como na organização dos dados. Para as questões abertas, foi utilizada a análise de conteúdo temático categorial, ${ }^{(15)}$ na qual a construção de categorias foi similar as variáveis de análise.

Este estudo foi apreciado e aprovado pelo Comitê de Ética em Pesquisa da Universidade do Estado do Rio de Janeiro, sob parecer de número 2.543.278. Após a aprovação ética, os participantes foram convidados a responder a pesquisa por meio de uma mensagem via e-mail na qual continha um endereço eletrônico que os dirigia ao instrumento. Todo o processo foi anônimo, já que os participantes não precisaram em nenhum momento realizar qualquer tipo de identificação pessoal no envio de dados, estando as informações vinculadas apenas ao e-mail indicado por eles no ato da assinatura do Termo de Consentimento Livre e Esclarecido (TCLE), que também foi preenchido de maneira virtual. Os participantes tiveram acesso aos contatos dos pesquisadores, bem como do comitê de ética que realizou a apreciação do projeto. Foi esclarecido que eventuais dúvidas sobre participação poderiam ser retiradas a qualquer momento e, a desistência do participante não implicaria em qualquer prejuízo, seja para pesquisa ou para o próprio participante.

$\mathrm{O}$ banco de dados gerado pelas respostas foi compartilhado apenas entre os pesquisadores e será extinto após cinco anos da coleta das informações, conforme legislação brasileira em vigor. Ressalta-se que este estudo atende a todas as diretrizes e normas de pesquisas envolvendo seres humanos da Resolução nº 466/2012. ${ }^{\text {(16) }}$

\section{Resultados}

Foram respondidos um total de 37 questionários online por enfermeiros residentes $(56,76 \%)$ e egressos $(43,24 \%)$ do Curso de Especialização na Modalidade Residência em Enfermagem em ESF, um total de $34(91,89 \%)$ questionários respondidos por mulheres e $3(8,1 \%)$ respondidos por homens. Desses 37 enfermeiros participantes da pesquisa $27(72,97 \%)$ tem a idade acima de 25 anos. Identifica-se também que a maior parte dos participantes se autodeclaram pardos $(37,84 \%)$ e brancos $(37,84 \%)$. A Tabela 1 traz esses dados de forma clara. 
Tabela 1. Distribuição dos participantes segundo a faixa etária, o sexo e a etnia. Rio de Janeiro, 2019

\begin{tabular}{|c|c|c|c|c|c|c|}
\hline \multirow[t]{2}{*}{ Variáveis } & \multicolumn{2}{|c|}{ Mulheres } & \multicolumn{2}{|c|}{ Homens } & \multicolumn{2}{|c|}{ Total } \\
\hline & f & $\%$ & $\mathbf{f}$ & $\%$ & $\mathbf{F}$ & $\%$ \\
\hline \multicolumn{7}{|l|}{ Faixa etária } \\
\hline De 21 a 23 anos incompletos & 4 & 10.81 & - & - & 4 & 10.81 \\
\hline De 23 a 25 anos incompletos & 6 & 16.22 & - & - & 6 & 16.22 \\
\hline Acima de 25 & 24 & 64.86 & 3 & 8.11 & 27 & 72.97 \\
\hline TOTAL & 34 & 91.89 & 3 & 8.11 & 37 & 100.00 \\
\hline \multicolumn{7}{|l|}{ Etnia } \\
\hline Pardo & 13 & 35.14 & 1 & 2.70 & 14 & 37.84 \\
\hline Branco & 13 & 35.14 & 1 & 2.70 & 14 & 37.84 \\
\hline Preto & 8 & 21.62 & 1 & 2.70 & 9 & 24.32 \\
\hline Cafuzo & - & - & - & - & - & - \\
\hline Cabloco & - & - & - & - & - & - \\
\hline Amarelo (Oriental) & - & - & - & - & - & - \\
\hline Indígena & - & - & - & - & - & - \\
\hline TOTAL & 34 & 91.89 & 3 & $8.11 \%$ & 37 & 100.00 \\
\hline
\end{tabular}

Fonte: Elaboração própria (2020)

Em relação ao tempo de formado dos participantes da pesquisa e a universidade de origem dos participantes, percebeu-se que $56,76 \%$ foram formados em universidades públicas e que apenas $13 \%$ da amostra possuem mais de 5 anos de formado.

Observou-se que $62,16 \%$ dos participantes da pesquisa já atenderam algum paciente com deficiência auditiva em sua Unidade Básica de Saúde (UBS) e outros, contudo, $100 \%$ dos participantes afirmaram não existir em sua unidade nenhum guia ou manual de orientação para auxiliá-los neste atendimento.

Em relação ao preparo da unidade para o atendimento, $40 \%$ dos enfermeiros informaram que há o reconhecimento institucional da demanda reprimida de usuários com deficiência auditiva no território, $83 \%$ afirmam a existência de atividades de promoção à saúde que asseguram a inclusão. Em relação à existência de acesso adequado para receber paciente com deficiência auditiva, para $49 \%$ dos participantes esta é inexistente e apenas $65 \%$ dos enfermeiros relataram a percepção e atendimento parcial das necessidades específicas das pessoas com deficiência auditiva por parte da equipe de saúde, sento o atendimento pleno um quantitativo inferior a $19 \%$.

Apenas $11 \%$ dos participantes relatam a existência de recursos na UBS que garantem a equidade no atendimento à pessoa com deficiência; Destes $50 \%$ relataram a presença de elevadores/rampas; $25 \%$ relataram a existência de transporte disponível para os dias de exames e consultas e outro participante relatou o atendimento domiciliar, através de visita como recurso utilizado pela sua unidade. 
Observou-se que para $92 \%$ participantes, a assistência à pessoa com deficiência auditiva oferecida pelas unidades de ESF não se dá de forma efetiva. Sobre os motivos para tal déficit, a falta de capacitação profissional e a ausência de recursos materiais e humanos foram os principais motivos relatados. Para 75,68 \% dos participantes, os indivíduos com deficiência auditiva receberam conteúdos insuficientes nas ações educativas quando comparados a população sem deficiência.

Em relação ao cuidado do enfermeiro voltado ao indivíduo com deficiência auditiva, apenas $4,35 \%$ dos participantes afirmaram não terem tido dificuldades para realizar as atividades de cuidado e, a linguagem foi considerada um grande dificultador para 34,78\% dos participantes os quais referiram que as ações educativas foram as mais prejudicadas.

Em relação a autoavaliação do enfermeiro relacionado ao cuidado a pessoa com deficiência auditiva, observa-se que $27 \%$ dos participantes indicam estarem totalmente despreparados para este atendimento, mesmo já sendo enfermeiros em processo de finalização da residência. Os demais participantes referiram estar parcialmente preparados para o cuidado, entretanto, tal preparo muitas vezes se deu por vivências pessoais com amigos e familiares surdos e, a realização de cursos específicos foi uma realidade observada em apenas $21 \%$ da amostra. Ressalta-se ainda que apenas $16 \%$ dos participantes referiram a residência como recurso de capacitação para o cuidado a pessoas com deficiência auditiva.

Todos os participantes referiram a emergente necessidade de uma estratégia educativa para qualificação específica ao cuidado à pessoa com deficiência auditiva. As estratégias citadas foram diversas, entretanto concentravam a responsabilidade no ensino de graduação, seja por disciplinas (44 \%) ou cursos e projetos de extensão universitária (45 \%).

\section{Discussão}

A residência é um espaço de formação e qualificação teórico-prático, no qual os alunos apontam grandes expectativas sobre este cenário, principalmente na ampliação da resolubilidade em serviço, ou seja, da capacidade de gerir, minimizar ou extinguir problemas, entretanto, este é um grande desafio, visto que os dois anos de prática nem sempre conseguem abarcar toda a complexidade do serviço, principalmente, quando se trata da saúde da família. ${ }^{(17)}$

Apesar do enfermeiro poder ingressar em uma residência a qualquer momento de sua trajetória profissional, no Brasil estas são fortemente associadas ao primeiro vínculo empregatício, sendo a primeira opção para jovens graduados. Assim, pesquisas que versam sobre o perfil de enfermeiros egressos da residência apontam para indivíduos do sexo feminino, com poucos anos de formação e ainda muito jovens. ${ }^{(18)}$

Não obstante a isso, ressalta-se que a Política Nacional de Saúde da Pessoa com Deficiência define, como propósitos gerais, um amplo leque de possibilidades que vai da prevenção de agravos à proteção da saúde, passando pela reabilitação; proteger a saúde da pessoa com deficiência; reabilitar a pessoa com deficiência na sua capacidade funcional e desempenho humano, contribuindo para a sua inclusão em todas as esferas da vida social. ${ }^{\text {(14) }}$ Em âmbito mundial, é necessário lembrar o compromisso da Agenda 2030, cujo objetivo n³ prevê a realização de atividades que promovam a saúde e assegurem o bem-estar para pessoas de todas as idades, incluindo aquelas com deficiências. ${ }^{(19)}$

Para a implementação da Rede de cuidados à saúde da pessoa com deficiência, fazse necessária a qualificação dos profissionais, bem como o comprometimento de gestores na disposição de ações, serviços e parcerias que consigam suprir as necessidades de saúde deste 
público-alvo. ${ }^{(20,21)}$ Apesar de serem estratégias intuitivas, tais ações ainda são um grande desafio para países em desenvolvimento, favorecendo a marginalização desta população e culminando na potencialização das limitações impostas ao cuidado integral. ${ }^{(22)}$

O incremento neste atendimento deve possuir foco na organização da Rede e na atenção integral à saúde contemplando as áreas de deficiência auditiva, física, visual, intelectual, transtornos do espectro do autismo e ostomias, ampliando a integração e articulação dos serviços de reabilitação com a rede de atenção primária e outros pontos de atenção especializada. Diante disso, faz-se necessário o reconhecimento acerca da situação de saúde da pessoa com deficiência atendida na ESF, sendo necessária a sensibilização dos profissionais de saúde sobre as necessidades gerais e específicas desta população, pois este é o primeiro passo para a implantação de um plano efetivo de cuidados. ${ }^{(23)}$

Contudo, as barreiras que atrapalham o atendimento às pessoas com deficiência na ESF são preocupantes, pois grande parte dos participantes observou que não possui acesso e meios para atender e promover a saúde em sua totalidade, sem que prejudicasse o processo de cuidar. Este resultado é compartilhado por outras pesquisas que indicam que o atendimento ao paciente com deficiência auditiva é um grande desafio à saúde pública mundial, sendo necessário um esforço interdisciplinar, intersetorial e criativo para garantir a integralidade do atendimento. ${ }^{(24-28)}$

Ressalta-se que a ESF constitui o nível de atenção primário que mais se aproxima dos indivíduos e das famílias e, por isso, destaca-se a importância de os profissionais de saúde tomar conhecimento e desenvolver programas de atenção que possam ir ao encontro às reais necessidades desta população.

Assim sendo, é essencial que primeiramente, o enfermeiro consiga realizar a comunicação com este público-alvo de forma efetiva, o que por si só já é considerado um grande desafio para os profissionais da saúde, visto o relato dos participantes sobre a ausência de preparo técnico para este fim. Neste sentido, o atendimento torna-se prejudicado, sofrendo grande impacto desde o estabelecimento do vínculo até a implementação do cuidado. ${ }^{(29)}$

Por isso, para que a assistência de enfermagem seja plenamente efetiva é necessário que se mantenha uma comunicação consciente, exigindo muito empenho para decodificar, decifrar e perceber o significado da mensagem que os pacientes estão querendo transmitir, pois somente assim é possível identificar as necessidades destes pacientes para que o profissional de enfermagem possa ajudá-los. ${ }^{(30)}$ Ressalta-se ainda que a inclusão destes indivíduos nas atividades da ESF é essencial pois é o contato inicial com o usuário e, em caso de necessidade, é o principal mecanismo de encaminhamentos à serviços especializados. ${ }^{(31)}$

Apesar dos profissionais adotarem estratégias não verbais no cuidado a pessoa com deficiência auditiva, as falhas na comunicação e na interação com esses pacientes caracterizam a necessidade de atenção e treinamento a fim de não comprometer o atendimento. ${ }^{(29)}$ Percebe-se que a assistência não se dá de forma efetiva, alinhando-se a falta de qualificação profissional e ausência de capacitação acerca da linguagem de sinais, que é considerada uma ferramenta decisiva na elaboração das formações discursivas dos surdos e a compreensão do seu discurso pelos profissionais da área de saúde propicia maior entendimento da constituição da identidade dos surdos, ponto fundamental para melhor qualidade dos serviços prestados. ${ }^{(32)}$

As dificuldades no atendimento foram relatadas por outros estudos, nos quais apontaram como possível solução, a inclusão de uma disciplina de linguagem de sinais na 
grade curricular dos profissionais de saúde, seja ela na formação básica, graduação ou em formações avançadas como em especializações. ${ }^{33,34)}$

Uma outra possibilidade para a inclusão destes usuários ao sistema de saúde é a incorporação de um tradutor que pudesse auxiliar a comunicação entre indivíduos e profissionais de saúde. É válido dizer que ações como esta demandam um alto investimento governamental, entretanto, podem igualar o acesso ao serviço de saúde entre indivíduos surdos e não surdos. ${ }^{(35)}$

Iniciativas como a criação de ambulatórios específicos para usuários com deficiência auditiva também foram consideradas bem-sucedidas. Na França esta iniciativa culminou no aumento do número de consultas anuais, chegando ao dobro do número original. ${ }^{(36)}$

A partir da percepção de que existe um déficit no cuidado a essa população, observase que grande parcela dos participantes percebem a necessidade de inclusão de recursos teórico-práticos na graduação em enfermagem, sendo evidenciado principalmente a necessidade de disciplina e de cursos de extensão que abordem o tema e qualifiquem estes profissionais para que estes prestem uma assistência efetiva à pessoa com deficiência auditiva.

\section{Conclusão}

Os enfermeiros identificam que a assistência à pessoa com deficiência auditiva não é eficiente, evidenciando, principalmente, a falta de capacitação profissional e falta de recursos humanos e materiais, pois grande parcela dos residentes e egressos percebem que não se sentem aptos a prestar esta assistência.

Esses achados reforçam a importância da capacitação para os profissionais já formados e de uma reorganização em relação às grades curriculares dos cursos de graduação em enfermagem, a fim de potencializar a resolutividade da assistência à pessoa com deficiência auditiva na ESF, com enfoque no acolhimento e no aprendizado de formas eficazes de comunicação.

É urgente a necessidade de se pensar em formas de qualificação dos profissionais de saúde e também na adaptação do ambiente de cuidado das unidades de saúde, garantindo a assistência a todos os cidadãos e em toda a rede de serviços, sobretudo, daqueles que por questões físicas ou psíquicas possuem condições que requeiram práticas ou estratégias diferenciadas de cuidado.

Ressalta-se ainda que no âmbito da residência em saúde da família, esta análise foi responsável por um diagnóstico conclusivo de insegurança, incapacidade de atendimento e despreparo da própria unidade de saúde, o que aponta para a necessidade de construção e reformulação de políticas públicas de educação permanente e de manutenção de ambientes institucionais de saúde amigáveis que possam garantir a estruturação de um sistema de atendimento resolutivo à esta população. 


\section{Referências Bibliográficas}

1. Santos AS, Portes AJF. Perceptions of deaf subjects about communication in Primary Health Care. Rev Lat Am Enfermagem [Internet]. 2019;27(27):e3127. Available from: https://dx.doi.org/10.1590\%2F1518-8345.2612.3127

2. World Health Organization (OMS). Deafness and hearing loss [Internet]. 2019 [cited 2021 Mar 17]. Available from: https://www.who.int/news-room/factsheets/detail/deafness-and-hearing-loss

3. Instituto Brasileiro de Geografia e Estatística. Censo Demográfico | IBGE - População residente por tipo de deficiência permanente [Internet]. 2010 [cited 2020 Dec 30]. Available from: https://www.ibge.gov.br/estatisticas/sociais/populacao/9662-censodemografico-2010.html?edicao=9749\&t=destaques

4. Simões A, Athias L, Botelho L. Panorama Nacional e Internacional da Produção de indicadores sociais. IBGE, editor. Rio de Janeiro; 2018. 352 p.

5. Nóbrega JD, Munguba MC, Pontes RJS. Atenção à saúde e surdez: desafios para implantação da rede de cuidados à pessoa com deficiência. Rev Bras em Promoção da Saúde [Internet]. 2017 [cited 2020 Dec 30];30(3):1-10. Available from: https://dx.doi.org/10.5020/18061230.2017.6176

6. Fuentes-López E, Fuente A. Access to healthcare for deaf people: a model from a middle-income country in Latin America. Rev Saude Publica [Internet]. 2020 [cited 2021 Mar 17];54:13. Available from: https://doi.org/10.11606/s15188787.2020054001864

7. Silva CT, Terra MG, Kruse MHL, Camponogara S, Xavier M da S. Residência multiprofissional como espaço intercessor para a educação permanente em saúde. Texto e Context Enferm [Internet]. 2016 [cited 2020 Dec 30];25(1):e2760014. Available from: http://dx.doi.org/10.1590/0104-0707201600002760014

8. Sena RR de, Grillo MJC, Pereira L d. Á, Belga SMMF, França BD, Freitas CP de. Educação permanente nos serviços de saúde: atividades educativas desenvolvidas no estado de Minas Gerais, Brasil. Rev Gaúcha Enferm [Internet]. 2017 [cited 2020 Jul 8];38(2):e64031. Available from: https://doi.org/10.1590/1983-1447.2017.02.64031

9. Ministério da Saúde (BR). Politica Nacional de Atenção Básica. Portaria n ${ }^{0}$ 2.436, de 21 de setembro de 2017 Brasília; 2017.

10. Schniedewind E, Lindsay RP, Snow S. Comparison of Access to Primary Care Medical and Dental Appointments Between Simulated Patients Who Were Deaf and Patients Who Could Hear. JAMA Netw Open [Internet]. 2021 Jan 21 [cited 2021 Jun 2];4(1):e2032207. Available from: https://jamanetwork.com/journals/jamanetworkopen/fullarticle/2775364 
11. Emond A, Ridd M, Sutherland H, Allsop L, Alexander A, Kyle J. Access to primary care affects the health of Deaf people. Br J Gen Pract [Internet]. 2015 [cited 2021 Jun 2];65(631):95-6. Available from: https://doi.org/10.3399/bjgp15X683629

12. Santos FPDA, Acioli S, Rodrigues VP, Machado JC, Souza MS, Couto TA. Nurse care practices in the Family Health Strategy. Rev Bras Enferm [Internet]. 2016 [cited 2020 Dec 31];69(6):1124-31. Available from: http://dx.doi.org/10.1590/0034-7167-20160273

13. Ayala-Hernandez JM, Lopera-Escobar A, Cadavid-Mazo SY, Pulgarin-Ossa DA. The Interaction between the Healthcare Professional and the Deaf Person. Experiences from the Colombian Healthcare System. J Dev Phys Disabil [Internet]. Springer; 2021 [cited 2021 Mar 17];1-12. Available from: https://doi.org/10.1007/s10882-020-09784-4

14. Ministério da Saúde (BR). Política Nacional de Saúde da Pessoa com Deficiência. Departamento de Ações Programáticas Estratégicas. Brasilia; 2010. 24 p.

15. Oliveira DC. Análise de conteúdo temático-categorial: uma proposta de sistematização. Rev Enf UERJ [Internet]. 2008 [cited 2019 Jul 22];16(4):569-76. Available from: http://www.facenf.uerj.br/v16n4/v16n4a19.pdf

16. Brasil. Resolução No 466 de 16 de dezembro de 2012. Diretrizes e normas regulamentadoras de pesquisa envolvendo seres humanos. Brasília; 2012.

17. Pereira AL de F, Guimarães JCN, Nicácio MC, Batista DB dos S, Mouta RJO, Prata JA. Perceptions of nurse-midwives of their residency training and professional practice. Reme Rev Min Enferm [Internet]. 2018 [cited 2020 Dec 30];22:e-1107. Available from: https://dx.doi.org/10.5935/1415-2762.20180035

18. Mello de Carvalho DJ, Silva RMO, Fernandes JD, Cordeiro ALAO, Santos OMB dos, Silva LS da, et al. Egressos de residência em enfermagem e o mercado de trabalho. Rev Enferm UFPE line [Internet]. 2019 Jun 14 [cited 2021 Jun 3];13. Available from: https://doi.org/10.5205/1981-8963.2019.238381

19. United Nations. SDG Good Practices | Department of Economic and Social Affairs [Internet]. [cited 2021 Mar 17]. Available from: https://sdgs.un.org/sdg-good-practices

20. Dubow C, Garcia EL, Krug SBF, Beatriz S, Krug F. Percepções sobre a Rede de Cuidados à Pessoa com Deficiência em uma Região de Saúde. Saúde em Debate [Internet]. 2018 [cited 2020 Dec 30];42(117):455-67. Available from: https://dx.doi.org/10.1590/0103-1104201811709

21. Machado WCA, Pereira J de S, Schoeller SD, Júlio LC, Martins MMFP da S, Figueiredo NMA de. Comprehensiveness in the care network regarding the care of the disabled person. Texto Context - Enferm [Internet]. 2018 [cited 2020 Dec 30];37(3):e4480016. Available from: https://doi.org/10.1590/0104-07072018004480016 
22. Kuenburg A, Fellinger P, Fellinger J. Health Care Access Among Deaf People. J Deaf Stud Deaf Educ [Internet]. 2016;21(1):1-10. Available from: https://doi.org/10.1093/deafed/env042

23. Condessa AM, Giordani JM do A, Neves M, Hugo FN, Hilgert JB. Barriers to and facilitators of communication to care for people with sensory disabilities in primary health care: a multilevel study. Rev Bras Epidemiol [Internet]. 2020 [cited 2020 Dec 30];23:E200074. Available from: https://dx.doi.org/10.1590/1980-549720200074

24. Reis V de SL, Santos AM dos. Conhecimento e experiência de profissionais das Equipes de Saúde da Família no atendimento a pessoas surdas. Rev CEFAC [Internet]. 2019 [cited 2020 Dec 31];21:e5418. Available from: https://doi.org/10.1590/1982$0216 / 20192115418$

25. Nascimento GB, Fortes LDO, Kessler TM. Estratégias de comunicação como dispositivo para o atendimento humanizado em saúde da pessoa surda. Saúde (Santa Maria) [Internet]. 2015 [cited 2020 Dec 31];41(2):241-50. Available from: https://doi.org/10.5902/2236583415121

26. Orrie S, Motsohi T. Challenges experienced by healthcare workers in managing patients with hearing impairment at a primary health care setting: a descriptive case study. South African Fam Pract [Internet]. 2018 [cited 2020 Dec 31];60(6):207-11. Available from: https://dx.doi.org/10.1080/20786190.2018.1507566

27. Sousa EM de, Almeida MAPT. Atendimento ao surdo na atenção básica: perspectiva da equipe multidisciplinar. Id Line Rev Psicol [Internet]. 2016 [cited 2020 Dec 31];10(33):72-82. Available from: https://dx.doi.org/10.14295/idonline.v10i33.589

28. Sanz Lledó A, García Vallejo R. Percepción de la atención sanitaria recibida en Atención Primaria por personas con discapacidad auditiva. Un estudio cualitativo. Rev Rol Enfermería [Internet]. 2019 [cited 2021 Mar 17];42(6):408-12. Available from: https://medes.com/publication/145358

29. Soares IP, Messias de Lima EM, Melo dos Santos AC, Ferreira CB. How do i talk to you? The communication of the nurse with the deaf user. Rev Baiana Enferm [Internet]. 2018 Aug 24;32:e25978. Available from: http://dx.doi.org/10.18471/rbe.v32.25978

30. Rodrigues SCM, Damião GC. Virtual Environment: Assistance in nursing care for the deaf based on the protocol of Primary care. Rev da Esc Enferm da USP [Internet]. 2014 [cited 2020 Dec 31];48(4):731-8. Available from: https://dx.doi.org/10.1590/S0080623420140000400022

31. Hashemi G, Wickenden M, Bright T, Kuper H. Barriers to accessing primary healthcare services for people with disabilities in low and middle-income countries, a Metasynthesis of qualitative studies. Disabil Rehabil [Internet]. 2020; Available from: https://doi.org/10.1080/09638288.2020.1817984

32. Santos IB dos, Marques JM, Berberian AP, Massi GA de A, Tonocchi R de C, Guarinello AC. Qualit y of life of deaf sign language users in the south of brazil. Saúde 
e Pesqui [Internet]. 2020 [cited 2020 Dec 31];13(2):295-307. Available from: https://doi.org/10.17765/2176-9206.2020v13n2p295-307

33. Sanches ICB, Bispo LP, Santos CH da S, França LS, Vieira SNS. O papel do enfermeiro frente ao paciente surdo. Rev Enferm UFPE line [Internet]. 2019 [cited 2020 Dec 31];13(3):858-62. Available from: https://doi.org/10.5205/1981-8963v13i03a238964p858-862-2019

34. França EG, Pontes MA, Costa GMC, França ISX. Dificuldades de profissionais na atenção à saúde da pessoa com surdez severa. Cienc y Enferm [Internet]. 2016 [cited 2020 Dec 31];22(3):107-16. Available from: http://dx.doi.org/10.4067/S071795532016000300107

35. Zulu T, Heap M, Sinanovic E. The cost and utilisation patterns of a pilot sign language interpreter service for primary health care services in South Africa. PLoS One [Internet]. 2017;12(12):e0189983. Available from: https://doi.org/10.1371/journal.pone.0189983

36. Amoros T, Bonnefond H, Martinez C, Charles R. Un dispositif ambulatoire pour la santé des Sourds en soins primaires. Sante Publique (Paris) [Internet]. 2014 [cited 2021 Jun 3];26(2):205. Available from: http://www.cairn.info/revue-sante-publique-2014-2page-205.htm

Participação dos autores: a) Planejamento e concepção do trabalho; b) Coleta de dados; c) Análise e interpretação de dados; d) Redação do manuscrito; e) Revisão crítica do manuscrito.

K. D. S. D. S. contribuiu em a, b, c, d; L. P. S. S. em a, b, c, d; M. G. D. A. F. em a, c, d, e; P. S. B. em a, c, d, e; P. C. D. S. T. em a, e; L. V. V. A. em a, e.

Editora científica responsável: Dra. Natalie Figueredo 